\title{
Phosphatidyl serine externalization in different age groups of mouse erythrocytes in response to agents that induce anemia
}

\begin{abstract}
Apoptosis in nucleated cells indicate the start of the programmed cell death. For erythrocytes that lack nucleus, corresponding changes are termed eryptosis and a prime change associated with eryptosis is the externalization of Phosphatidyl serine (PS) on erythrocyte membrane. It is not possible to assess PS externalization in vivo since PS + erythrocytes are immediately cleared in vivo by macrophages. Eryptosis may be an important component of the mode of action of agents that induce anemia. In this study, we have examined the PS externalization response to several agents known to induce anemia. The test agents included poly-dispersed acid functionalized carbon nanotubes (AF-SWCNTs), carboxylated Nanodiamonds (cNDs), cadmium chloride, paraquat dichloride and anti-erythrocyte antibodies (AEA). Mouse blood derived erythrocytes were incubated for 1 and 3 hours with various agents, and Phosphatidyl serine (PS) externalization on erythrocyte membrane studied flow cytometrically after staining with annexin V. All test-agents induced PS externalization in erythrocytes, the maximum effect being that of cadmium chloride. Further, PS externalization was also studied separately in young ( 0 to 10 days in blood circulation), intermediate (age 10 to 40 days in circulation) and old (age above 40 days in circulation) erythrocytes. Using the double in vivo biotinylation (DIB) technique, the three age groups of erythrocytes could be gated flow cytometrically and PS externalization compared in different age groups. In all cases, maximum PS externalization was observed in young group of erythrocyte even though quantitatively the maximum effect was seen in case of Cadmium chloride and AF-SWCNTs. Correlation of these in vitro results with the erythrocyte turnover data in mice with induced anemia has been discussed.
\end{abstract}

Keywords: erythrocytes, biotin, eryptosis, annexin V, AF-SWCNTs, cadmium, paraquat, Anti-mouse
Volume 6 Issue 4 - 2018

\section{Md Babu Mia, Rajiv K Saxena}

Department of Life Sciences and Biotechnology, South Asian University, India

Correspondence: Rajiv K Saxena, Faculty of Life Sciences and Biotechnology, South Asian University, Akbar Bhawan, Chanakyapuri, New Delhi I I002I, India, Tel 91-991004824I, Email rksaxena@sau.int, rajivksaxena@hotmail.com

Received: June 06, 2018 | Published: July 30, 2018
Abbreviations: PS, phosphatidyl serine; cNDs, carboxylated nanodiamonds; AEA, anti-erythrocyte antibodies; DIB, double in vivo biotinylation; SOP, standard operating procedures; SAv-APC, streptavidin-allophycocyanin; PE, phycoerythrin; SWCNT: single walled carbon nanotubes; DMF, dimethylformamide; FBS, fetal bovine serum; BXN, biotin-X-NHS ester

\section{Introduction}

Erythrocytes constitute around $99.9 \%$ of total blood cells excluding platelets with the half-life of around 60 and 120days in mice and human respectively. In general, the blood erythrocyte count remains steady and is dependent upon a fine balance between rate of destruction of erythrocytes and the rate of formation of fresh erythrocytes in bone marrow. Previous studies from our laboratory have attempted to gain an insight into the mechanism of induction of anemia in response to a variety of anemiagenic agents like poly-dispersed acid functionalized carbon nanotubes (AF-SWCNTs), carboxylated Nanodiamonds (cNDs), cadmium chloride, paraquat dichloride and anti-erythrocyte antibodies (AEA). ${ }^{1-6}$ Anemia may occur due to a variety of factors that may include depressed erythropoiesis, enhanced elimination of erythrocytes from circulation, induction of apoptosis (termed eryptosis for erythrocytes) and cytotoxicity of the anemia inducing agents, phagocytosis of the erythrocytes and expression of "don'teat-me" signals like CD47 on erythrocyte sub-populations. ${ }^{7-9}$ Role of eryptosis as a factor in the induction of anemia is not clear since the erythrocytes that show Phosphatidyl Serine (PS) extrusion as a marker of eryptosis, are rapidly cleared in vivo by phagocytosis and do not accumulate in blood.

In the present communication, we have compared the in vitro induction of eryptosis in whole erythrocyte population as well as in different age groups of erythrocytes. Erythrocytes in blood circulation belonging to different age groups could be identified by using a recently developed double in vivo biotinylation (DIB) technique and PS externalization in each age group of erythrocytes could be examined in vitro. These results have provided information about the role of eryptosis in the induction of anemia by different anemiagenic agents. Significant yet variable eryptotic response was seen in blood erythrocytes by all test agents. Also, in all cases, the eryptotic response was most pronounced in the old age group of erythrocytes and was relatively low in the youngest erythrocytes, being minimal in reticulocytes. Implications of these findings in the mechanisms of induction of anemia have been discussed.

\section{Materials and methods}

\section{Animals}

Inbred C57BL/6 male mice (8-12weeks old, 20-25g body weight) and Wister female rat (8weeks old, 250-300g body weight) 
were used in this study. Animals were obtained from the National Institute of Nutrition (NIN), Hyderabad and maintained in the microbe-free environment in the animal house facility at South Asian University, New Delhi. Animals were given food and water at ad libitum and always kept in positive pressure air-conditioned units. All experimental protocols were conducted strictly in compliance with the Standard Operating Procedures (SOP) for Institutional Animal Ethics Committee (IAEC) of the CPCSEA (Committee for Control and Supervision on Experiments on Animals), Ministry of Environment, Forest and Climate Change, Government of India. The study was duly approved by Institutional Animal Ethics Committee of South Asian University. All mice were randomly assigned to experimental groups.

\section{Reagents and other supplies}

Biotin-X-NHS (N-hydroxysuccinimide ester of biotin) was obtained from Sigma Aldrich (St. Louis, MO, USA). StreptavidinAllophycocyanin (SAv-APC), anti-mouse CD71-Phycoerythrin (PE) monoclonal antibodies, anti-mouse CD16/CD32 purified and Annexin V-FITC recombinant proteins were purchased from Biolegend or eBioscience or BD BioScience (USA). Single Walled Carbon Nanotubes (SWCNT) was from Sigma Aldrich, USA, carboxylated Nanodiamonds (average size $15 \mathrm{~nm}$, charge- $45 \mathrm{mV}$ ) were from Adamas nanotechnologies, USA. Cadmium Chloride was obtained from Fisher Scientific. RPMI culture medium, HEPES, Dimethylformamide (DMF), CPD (Citrate Phosphate Dextrose) and Paraquat dichloride hydrate, was procured from Sigma Aldrich. Fetal bovine serum (FBS) was obtained from Hyclone (South Logan, UT, USA). All other chemicals were purchased locally and were of analytical grade

\section{Generation of rat anti-mouse erythrocyte antibody serum}

Mouse erythrocytes $\left(2 \times 10^{8}\right.$ in $\left.200 \mu \mathrm{PBS}\right)$ were injected intraperitoneally into Wister female rat (two injections on day 0 and day 7 ). After four days of the second injection, rat blood was collected from tail vein and serum isolated as described before. ${ }^{5}$ The potency of ratanti-mouse erythrocyte antiserum was assessed by hemagglutination assay in which the antiserum agglutinated mouse erythrocytes till a dilution of $1: 128$.

\section{Acid functionalization of carbon nanotubes}

Acid functionalization of single walled carbon nanotubes SWCNTs were done by treatment with a mixture of concentrated $\mathrm{HNO}_{3}$ and $\mathrm{H}_{2} \mathrm{SO}_{4}$ in a high-pressure microwave digester. The procedure for acid-functionalization of SWCNTs and the characterization of the AF-SWCNTs have been described before. ${ }^{6,7}$ Acid-functionalized SWCNTs (AF-SWCNTs) had a charge of $-48 \mathrm{mV}$ and an average size of $232 \mathrm{~nm}$ as measured on a Zeta-Sizer equipment.

\section{Double in vivo biotinylation (DIB) technique}

Rationale and procedure for Double in vivo biotinylation (DIB) technique have been published before. ${ }^{1-9}$ In short, mice were administrated intra venously Biotin-X-NHS Ester (BXN) (1 mg BXN dissolved in $20 \mu 1$ of DMF and $250 \mu$ l of PBS) daily for three days followed by a 30 -day rest. A second low dose biotinylation was done after 30 days by i.v. administration of $0.6 \mathrm{mg}$ of BXN dissolved in $12 \mu \mathrm{l}$ of DMF and $250 \mu 1$ of PBS. After 10 days of the second biotinylation step, erythrocytes were stained with avidin, and anti-CD71 (reticulocyte marker) and analyzed flow cytometrically as described before. ${ }^{2,8,9}$ The technique allowed us to gate on four subpopulations of erythrocyte (young, intermediate and old groups of erythrocytes as well as on reticulocytes). Protocol for gating erythrocytes into young, intermediate and old age groups as well as for reticulocytes has been standardized and described elsewhere. ${ }^{2,6,8,9}$ Briefly, erythrocytes were washed with PBS and incubated and stained with Streptavidin$\mathrm{APC}$ and anti-mouse CD71 PE to enable flowcytometric gating for reticulocytes and erythrocytes of different age groups. A typical flow cytometry histogram showing the gating of the four erythrocyte subpopulations are shown in Figure 1. For phosphatidyl serine (PS) externalization study, erythrocytes were incubated with Annexin V-FITC in annexin binding buffer as described before. ${ }^{13}$ All Flow cytometry study was performed by FACVerse and analyzed using FACSuite software.

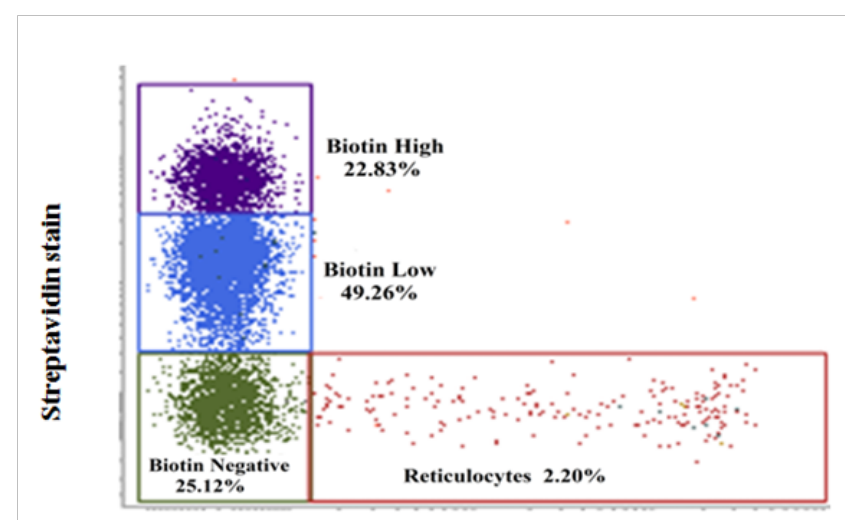

Anti-mouse CD71

Figure I Gating for reticulocytes and erythrocytes of different age groups in DIB technique. C57BL/6 mice were administered three daily i.v. doses of I mg BXN (| ${ }^{\text {st }}$ step of biotinylation). After 30 days of rest, a single additional $0.6 \mathrm{mg} B X N$ dose ( $2^{\text {nd }}$ biotinylation step) was administrated. Erythrocytes were collected 10 days after $2^{\text {nd }}$ dose of BXN, washed with PBS, and stained with streptavidin and anti-mouse CD7I antibody to differentiate and identify different age cohorts of erythrocytes based on the intensity of streptavidin and anti-mouse CD7I binding with erythrocytes. This is an illustrative Figure. ${ }^{2,9}$

\section{Induction of PS externalization}

Mouse blood derived erythrocytes were incubated in 48 well plate, with or without AF-SWCNTs $(100 \mu \mathrm{g} / \mathrm{ml})$, cNDs $(100 \mu \mathrm{g} / \mathrm{ml})$, $\mathrm{CdCl}_{2}(1000 \mathrm{ppm})$, paraquat $(50 \mu \mathrm{g} / \mathrm{ml})$ and rat anti-mouse serum $(1: 50$ dilution). After 1 and 3hours of incubation, cells were stained with annexin- $\mathrm{V}$ and analyzed flowcytometrically as described before. ${ }^{2,8,13}$

\section{Statistical analysis}

All experiments were repeated three times. Representative data has been shown in all figures where Mean \pm SEM values were derived from data obtained with three individual preparations of blood erythrocytes from individual mice. All graphs were prepared by Sigma Plot software. Statistical analysis was performed by Student's t-test and $\mathrm{p}<0.05$ was accepted as statistically significant.

\section{Result}

\section{Induction of PS externalization in mouse erythrocytes by carbon nanoparticles, heavy metal ions, paraquat and anti-erythrocyte antibodies}

To compare the induction of PS externalization in response to 
carbon nano particles, cadmium ions, paraquat dichloride and antierythrocyte antibodies (AEA), mouse erythrocytes were incubated with AF-SWCNTs $(100 \mu \mathrm{g} / \mathrm{ml})$, cNDs $(100 \mu \mathrm{g} / \mathrm{ml}), \mathrm{CdCl}_{2}(1000 \mathrm{ppm})$, paraquat $(50 \mu \mathrm{g} / \mathrm{ml})$ and AEA (1:50 dilution) for 1 and 3 hours and stained with annexin $\mathrm{V}$ to assess the externalization of phosphatidyl serine on erythrocyte membrane. Results of a representative experiment are shown in Figure 2. Top panel shows the results of annexin $\mathrm{V}$ staining of control untreated erythrocytes indicating that in control erythrocytes more than $99 \%$ of the cells were negative for annexin V staining. These control histograms were used for setting the flow cytometric gate above which PS externalization would be indicated. For cND treated erythrocytes, about $12.85 \%$ cells were annexin $\mathrm{V}$ positive after one hour of treatment whereas this percentage was $19.12 \%$ after 3 hours of treatment with nanodiamonds. PS externalization was also seen for treatments with AF-SWCNTs, $\mathrm{CdCl}_{2}$, Paraquat dichloride as well as anti-erythrocyte antibodies, and in general the Phosphatidyl externalization increased with time. In case of cadmium chloride, increase in the eryptotic response on 3-hour time was relatively high, increasing from $5.56 \%$ at $1 \mathrm{~h}$ to $42.88 \%$ at $3 \mathrm{~h}$. These results show that all agents we examined could induce significant externalization of Phosphatidyl serine in erythrocytes; cadmium chloride being the most potent in this regard, followed by nanodiamonds. Lowest PS externalization was seen in the case of AF-SWCNTs and anti-erythrocyte antiserum, but in these cases too, significant PS externalization response was observed.

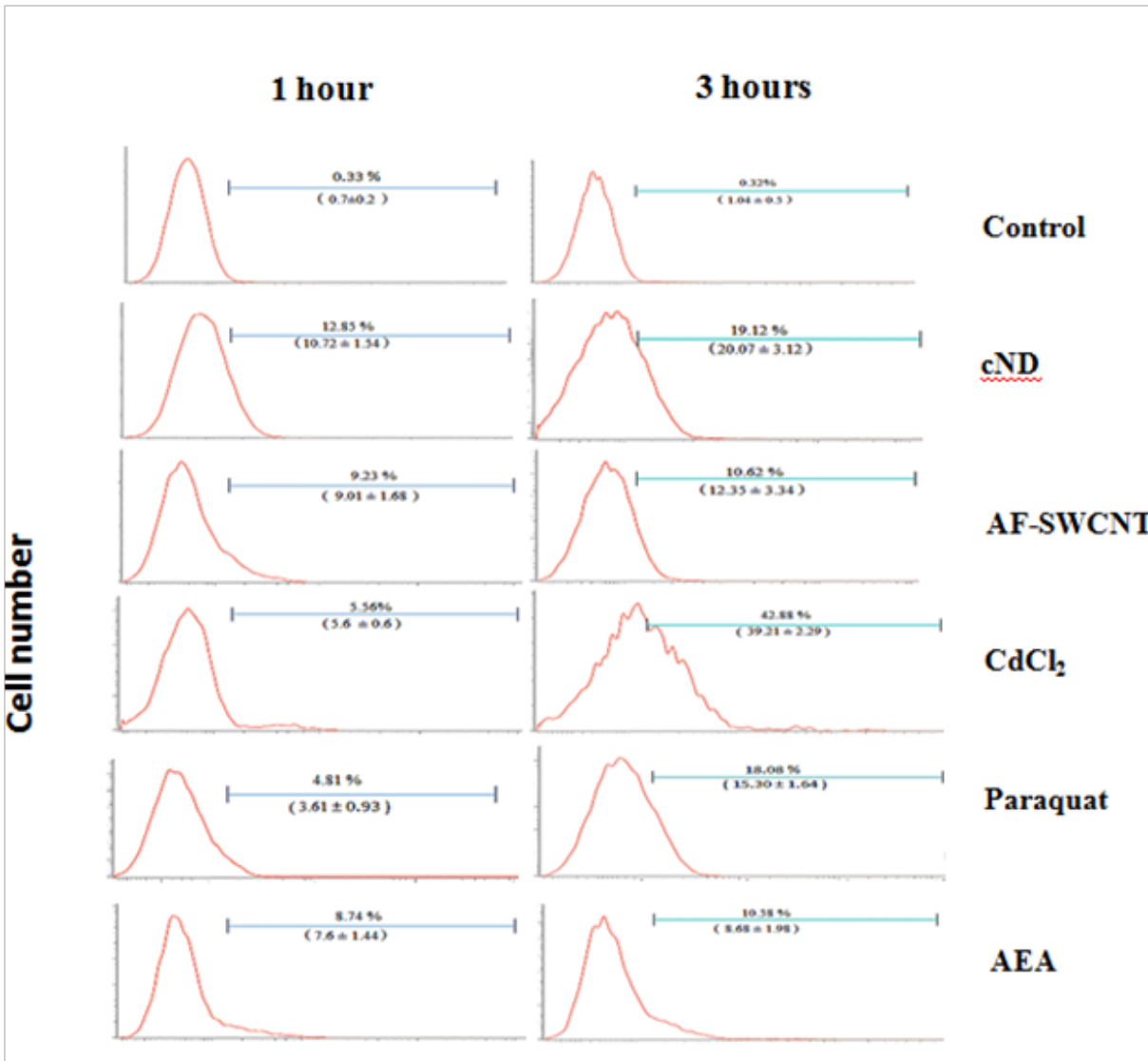

Annexin-V stain

Figure 2 PS externalization on erythrocytes in response to various test agents. Mouse erythrocytes (Ix|06 per ml in RPMI medium $+10 \%$ FCS) were incubated

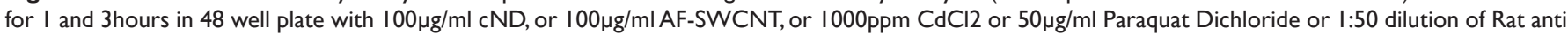
mouse serum. After the culture, erythrocytes were stained with Annexin V-FITC and CD7I-PE and analyzed on flow cytometer.Values in brackets denote the mean response + SEM of data obtained with erythrocyte preparations from three individual mice.

Dependence of the eryptotic response on the age of erythrocytes in blood circulation

Blood derived erythrocytes constitute a mixture of erythrocytes that have spent various lengths of time in blood circulation. It was of interest to determine if the age of erythrocytes in circulation has any bearing on the eryptotic response to various test agents. We have developed a new technique (double in vivo biotinylation technique or the DIB technique) that allows us to flow cytometrically gate on erythrocytes of various age groups. ${ }^{1-9}$ In the specific protocol we used for the present experiments, we could specifically gate on erythrocytes that belonged to young ( 0 to 10 days in blood circulation), intermediate (age 10 to 40 days in circulation) and old (age above 40 days in circulation) and compare the eryptotic response in the three age groups. In addition, we could also gate on reticulocytes and eryptotic response in reticulocytes could also be assessed.

Erythrocytes were obtained from mice subjected to double-in vivobiotinylation (DIB) protocol and incubated for 1 or 3hours with the four test agents to compare the eryptotic response in reticulocytes as well as erythrocytes belonging to different age groups. Results of these experiments are summarized in Figure 3. In case of nanodiamond 
(Figure 3, Panel A) treated erythrocytes, PS externalization was comparable (about $8 \%$ ) in young, intermediate as well as old groups of erythrocytes at 1 hour time point. At 3 hour time point, eryptotic response increased in all erythrocyte groups, but old erythrocytes had significantly more PS externalization as compared to young and intermediate age groups of erythrocytes.

In case of AF-SWCNTs (Figure 3, Panel B) PS externalization in young and intermediate groups of erythrocytes were comparable (about 5\% and 10\% eryptosis at 1 and 3 hours' time points respectively) but in old age group of erythrocytes, the response was significantly higher, being about $40 \%$ and $20 \%$ at 1 and 3hours respectively. This was the only case where PS externalization was more at $1 \mathrm{~h}$ time point than the $3 \mathrm{~h}$ time point. The reason could be that at later time point, many erythrocytes could have lysed and disappeared from the culture.

PS externalization induced by cadmium chloride was low at one-hour time point but was markedly elevated at 3hour time point where the percentage of eryptotic cells increased from young $(25 \%)$ to intermediate $(30 \%)$ and to old age groups $(45 \%)$ of erythrocytes (Figure 3, panel C).Anti-mouse erythrocyte antiserum also induced PS externalization that too was highest in the old group of erythrocytes at both time points (Figure 3, panel E). Impact of paraquat on all age groups of erythrocytes was almost similar and induced increased PS externalization (about $12 \%$ ) from 1 hour to 3 hours' time point (Figure 3, panel D).

\section{PS externalization in reticulocytes}

Reticulocytes are the youngest subgroup of young erythrocytes and the percentage of reticulocytes that showed PS externalization was in general very low as compared to young, intermediate and old groups of erythrocytes in all cases (Figure 3, all panels). Highest eryptosis was seen with cadmium chloride and that too was less than 3\% (Figure 3, panel C).

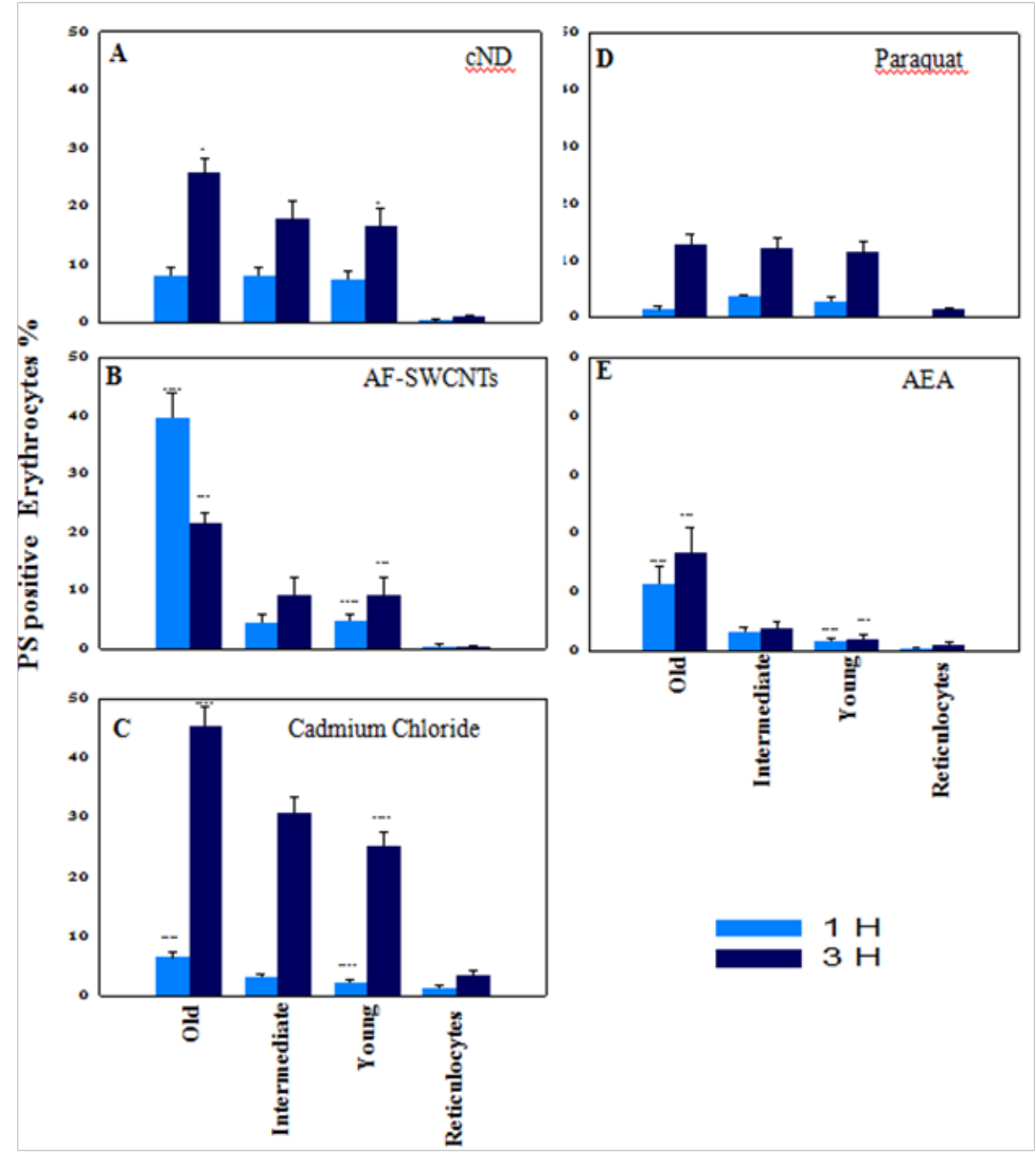

Figure 3 PS externalization on erythrocytes of different age groups and reticulocytes in response to various test agents. Blood was collected from double in vivo biotinylated mice from tail vein and erythrocytes isolated. Erythrocytes (I 106 per $\mathrm{ml}$ in RPMI medium $+10 \% \mathrm{FCS})$ were incubated for I and 3 hours in 48

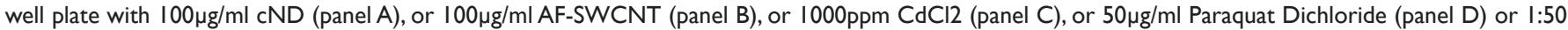
dilution of Rat anti mouse serum (panel E).After the culture, all control and treated erythrocyte preparations were stained with Streptavidin-APC and CD7I-PE to delineate different age groups of erythrocytes and reticulocytes (as shown in Figure I), and Annexin V-FITC to determine the PS externalization on different age groups of control and treated erythrocyte preparations (as in Figure 2), followed by flow cytometry. Each bar on the graph represents mean percentages of PS+ erythrocytes \pm SEM of data derived with three individual erythrocyte preparations from individual mice.

$*_{p}<0.05, * * p<0.01, * * * p<0.005$ and $* * * * p<0.001$ for comparison of the groups (Student $t$-test). 


\section{Discussion}

Anemia is defined by a significant decline in the blood erythrocyte count and hemoglobin levels. A decline in erythropoietic activity in bone marrow or an increased rate of destruction of erythrocytes in blood circulations, or both may result in anemic condition. ${ }^{10-12,14-16}$ Further, the rate of erythropoiesis is regulated by many factors like the levels of erythropoietin and other cytokines, damage to bone marrow by radiations or chemotherapy and availability of iron and its utilization etc. ${ }^{10,14,15}$ Rate of destruction of erythrocytes is likewise dependent on a large number of factors like presence of toxic agents, oxidative stress and expression of CD47 marker on erythrocytes. ${ }^{9,13,17}$ Using a newly developed double in vivo biotinylation technique, ${ }^{1-9}$ we have examined the changes in the erythrocyte turnover kinetics and erythropoietic activities in several models of anemia. ${ }^{1-9}$ In anemia induced by cadmium ions, paraquat and acute anemia following the administration of a single dose of AF-SWCNTs, we found an increase in the relative proportion of young erythrocytes in anemic mice that would be a reflection of preferential elimination of older erythrocytes. $3,4,7$ Interestingly, in autoimmune anemia and chronic anemia induced by repeated administration of AF-SWCNTs, we found a significant increase in the relative proportion of older erythrocytes in the blood. ${ }^{5,6}$ Elimination of erythrocytes follows the externalization of phosphatidyl serine that is recognized by macrophages and initiates phagocytosis. In the two types of anemia where young or old erythrocytes are preferably eliminated, it would follow that eryptotic changes also occur in the erythrocytes to be eliminated. It is not possible to study eryptotic changes in vivo, since PS externalization immediately results in the phagocytosis of the erythrocytes. In the present study therefore, we have studied the eryptotic changes resulting from exposure to $\mathrm{CdCl}_{2}$, paraquat, AF-SWCNTs, nano diamonds and antimouse erythrocyte antibodies in vitro.

Our results indicate that exposure (1 or 3hours) to all test agents in vitro induced PS externalization in mouse blood erythrocytes induced PS externalization in erythrocytes and the magnitude of the effect was $\mathrm{CdCl}_{2}>\mathrm{cND}>\mathrm{AF}-\mathrm{SWCNT}=$ Paraquat $>$ AEA. Maximum PS externalization $\left(43 \% \mathrm{PS}^{+}\right.$cells) was seen with 3 hour incubation with $\mathrm{CdCl}_{2}$ and minimum of (about $3 \% \mathrm{PS}^{+}$cells) for 1 hour exposure to paraquat. DIB technique was used to flow cytometrically gate on young (age in circulation $<10$ days), intermediate (age in circulation 10-40 days) and old (age in circulation $>40$ days) erythrocytes to compare PS externalization on these different age groups of erythrocytes. Our results indicated that in all cases, The PS externalization was seen in all age groups of erythrocytes but the maximum response to all test agents was seen with erythrocytes of the old group. Minimum variation in the PS externalization in age groups of erythrocytes was seen with paraquat where at the three-hour time point, PS externalization was almost similar in all age groups.

In anemia models where we had observed a significant increase in the relative proportion of young erythrocytes, a higher PS externalization response in old group of erythrocytes was expected, and we could see it in the case of $\mathrm{CdCl}_{2}$ and AF-SWCNTs. Carboxylated NDs belonging to carbon nanoparticle, behaved in a way similar to AF-SWCNTs. Though we have not studied cND induced anemia in mice, a report in literature indicates a toxic effect of nanodiamonds on erythrocytes. ${ }^{18}$ In case of AEA also, old erythrocytes had significantly higher PS externalization than the young and intermediate groups of erythrocytes. This was contrary to the results expected in view of a relative increase in the proportion of old erythrocytes in auto immune hemolytic anemia that we had previously reported. ${ }^{5}$ Increased old population would be expected to result from an increased elimination of young erythrocytes but we found a higher PS externalization in old population of erythrocytes. It is likely that factors other than the induction of PS externalization may come into play in case of antibody induced anemia. It is interesting to note that there is a reticulocytopenia and bone marrow aplasia associated with autoimmune hemolytic anemia $^{5}$ and a reduced supply of younger erythrocytes rather than elimination of PS externalized young erythrocytes may have been responsible for increased population of old erythrocytes in this model of anemia

\section{Conclusion}

We have found that all the agents that induce anemia in mice, also induce PS externalization in erythrocytes in vitro, but in all cases the maximum PS externalization occurs in the old group of erythrocytes indicating that this group of erythrocytes may be preferentially eliminated in vivo. In cases where younger erythrocytes seem to be eliminated in vivo model of anemia, factors other than PS externalization e.g. rate of erythropoiesis may be important factors in the induction of anemia

\section{Acknowledgments}

This work was supported by the Department of Science and Technology, Government of India, Nano-sciences Mission grant and JC Bose award to RKS. MBM received fellowship support from the South Asian University, New Delhi.

\section{Conflict of interest}

The author declares that there is no conflict of interest.

\section{References}

1. Khandelwal S, Saxena RK. Assessment of survival of aging erythrocyte in circulation and attendant changes in size and CD147 expression by a novel two step biotinylation method. Exp Gerontol. 2006;41(9):855-861.

2. Saxena RK, Bhardwaj N, Sachar S, et al. A double in vivo biotinylation technique for objective assessment of aging and clearance of mouse erythrocytes in blood circulation. Transfus Med Hemother. 2012;39(5):335-341.

3. Chatterjee S, Saxena RK. Preferential elimination of older erythrocytes in circulation and depressed bone marrow erythropoietic activity contribute to cadmium induced anemia in mice. PLoS One. 2015;10(7):e0132697.

4. Bhardwaj N, Saxena RK. Elimination of young erythrocytes from blood circulation and altered erythropoietic patterns during paraquat induced anemic phase in mice. PLoS One. 2014;9(6):e99364.

5. Chatterjee S, Bhardwaj N, Saxena RK. Identification of stages of erythroid differentiation in bone marrow and erythrocyte subpopulations in blood circulation that are preferentially lost in autoimmune hemolytic anemia in mouse. PLoS One. 2016;11(11):e0166878.

6. Bhardwaj N, Saxena RK. Selective loss of younger erythrocytes from blood circulation and changes in erythropoietic patterns in bone marrow and spleen in mouse anemia induced by poly-dispersed single-walled carbon nanotubes. Nanotoxicology. 2015;9(8):1032-1040.

7. Sachar S, Saxena RK. Cytotoxic effect of poly-dispersed single walled carbon nanotubes on erythrocytes in vitro and in vivo. PLoS One. 2011;6(7):e22032.

8. Chatterjee S, Saxena RK. Erythrocyte Turnover and Erythropoietic 
Patterns in Two Different Experimental Mouse Models of Anemia. In: Atta-ur-Rehman, editor. Frontiers in Clinical Drug Research: Hematology. UAE: Bentham Science Publishers; 2018. p. 220-240.

9. Rajiv K Saxena, Khandelwal S. Aging and destruction of erythrocytes in mice. Current Science. 2009;97:500-507.

10. Mohanty JG, Nagababu E, Rifkind JM. Red blood cell oxidative stress impairs oxygen delivery and induces red blood cell aging. Front Physiol. 2014;5:84.

11. Alaarg A, Schiffelers RM, Van Solinge WW, et al. Red blood cell vesiculation in hereditary hemolytic anemia. Front Physiol. 2013;4:365.

12. Meunier B, Loosveld M, Grados A, et al. Bone marrow erythrophagocytosis and reticulocytopenia in autoimmune haemolytic anaemia. Br J Haematol. 2017;177(3):346.

13. Khandelwal S, Saxena RK. A role of phosphatidylserine externalization in clearance of erythrocytes exposed to stress but not in eliminating aging populations of erythrocyte in mice. Exp Gerontol. 2008;43(8):764-770.

14. Lutz HU, Bogdanova A. Mechanisms tagging senescent red blood cells for clearance in healthy humans. Front Physiol. 2013;4:387.

15. Lodish H, Flygare J, Chou S. From stem cell to erythroblast: regulation of red cell production at multiple levels by multiple hormones. IUBMB Life. 2010;62(7):492-496.

16. Luo ST, Zhang DM, Qin Q, et al. The Promotion of Erythropoiesis via the Regulation of Reactive Oxygen Species by Lactic Acid. Sci Rep. 2017;7:38105.

17. Khandelwal S, van Rooijen N, Saxena RK. Reduced expression of CD47 during murine red blood cell (RBC) senescence and its role in RBC clearance from the circulation. Transfusion. 2007;47(9):1725-1732.

18. Puzyr AP, Neshumaev DA, Tarskikh SV, et al. Destruction of human blood cells in interaction with detonation nanodiamonds in experiments in vitro. Diamond and Related Materials. 2004:13(11-12):2020-2023. 